Para citar este artículo / To cite this article:

Sirvent Ramos, Ángeles. «Estado actual de la investigación en Literatura francesa y Género. Perspectiva desde España y Francia. A modo de introducción». En Feminismo/s, 34 (diciembre 2019): 13-42. Dosier monográfico: Estado actual de la investigación en Literatura francesa y Género: balance y nuevas perspectivas / État présent de la recherche en Littérature française et Genre: bilan et nouvelles perspectives, coord. Ángeles Sirvent Ramos, DOI: 10.14198/fem.2019.34.01

\title{
ESTADO ACTUAL DE LA INVESTIGACIÓN EN LITERATURA FRANCESA Y GÉNERO. PERSPECTIVA DESDE ESPAÑA Y FRANCIA. A MODO DE INTRODUCCIÓN
}

\section{CURRENT STATE OF RESEARCH IN FRENCH LITERATURE AND GENDER. PERSPECTIVES FROM SPAIN AND FRANCE. BY WAY OF INTRODUCTION}

\author{
Ángeles SIRVENT RAMOS \\ Universidad de Alicante, Alicante \\ https://orcid.org/0000-0001-7931-0658 \\ ma.sirvent@ua.es
}

Palabras clave: escritoras francesas; historia de la literatura; investigación con perspectiva de género; universidad española; coloquios de la AFUE.

Keywords: French women writers; History of literature; Gender research; Spanish university; Colloquiums of AFUE.

La conciencia de la desigualdad de género está arraigándose afortunadamente en nuestra sociedad, por mucho que una lastra histórica de dominación masculina sigue produciendo, lamentablemente, nefastas consecuencias.

La universidad, en tanto que institución del pensamiento reflexivo y racional del más alto nivel, así como la investigación que en ella, y desde ella, se realiza y se proyecta, originan debates constructivos y suplen la invisibilidad 
que, en distintos ámbitos de la historia de la cultura y la ciencia aplicada, la mujer ha padecido.

Las redes de investigación nacionales e internacionales de investigadoras e investigadores que atienden la perspectiva de género son cada vez más numerosas. La convocatoria de los proyectos europeos Horizonte 2020 la define claramente en una de sus modalidades.

Dicha perspectiva de género se contempla en las distintas convocatorias, en las ayudas a la realización de proyectos y organización de eventos científicos; no exento ello de cierta problemática y crítica cuando, con el objetivo de premiar la paridad, se penaliza a las redes, comisiones científicas, organizadoras o participantes mayoritariamente femeninas, lo que podría parecer una paradoja. Todo cambio implica reacción, y esperemos que esta política consiga el objetivo mayor, que es la inclusión progresiva de mujeres investigadoras en ámbitos todavía demasiado masculinizados. Pensemos pues, como decía Leibnitz, que los males particulares deben ser asumidos en pro de un bien general, aun cuando, como ironizaba el Candide de Voltaire, cuanto mayor sea el bien general, más habrá que soportar los males particulares.

En numerosas editoriales, tanto en España como en Francia, países objeto de nuestras perspectivas en este volumen, se van creando colecciones en las que el género y la visibilidad de las mujeres, ya sea en la creación o la investigación, son cada día más patentes.

Francia, sorprendentemente, se está mostrando más reacia a incluir en la universidad la perspectiva de género en sus estudios de licenciatura -dejando para los postgrados o doctorado la conciencia efectiva de la misma-, no potencia la inclusión de escritoras en las lecturas de bachillerato -hecho que Martine Reid, participante en este volumen, ha puesto de manifiesto en diversas ocasiones- o en los concursos docentes, e incluso muestra ciertas reservas al lenguaje inclusivo y a la feminización de términos como catedrática, paradójicamente patente incluso en universitarias no tan alejadas de los estudios de género. Aun con todo, hay que poner de relieve distintas iniciativas, como el ingente e importante trabajo de la SIEFAR (Société Internationale pour l'Étude des Femmes de l'Ancien Régime), que lleva a cabo una labor de innegable relevancia en pro de la visibilidad de las mujeres de pasados siglos, tanto desde el punto de vista socio-histórico como literario; iniciativas como las de la propia Martine Reid, creando la colección «Folio 2E», en la conocida 
editorial Gallimard, en donde a dicho módico precio se han publicado diferentes textos cortos de escritoras, favoreciendo su compra y su lectura.

Recordemos que en Francia se creó ya en 1973, gracias a Hélène Cixous, el «Centre de recherches en études féminines», ligado a Vincennes, la actual Université Paris 8 -denominado desde 2006 «Centre d'études féminines et d'études de genre», modificación altamente significativa-, y que en 1974 la propia Cixous pondría en funcionamiento el primer doctorado europeo en este ámbito: el doctorado pluridisciplinar en «Études féminines». Recordar su trayectoria no es objeto de estas líneas, pero remitimos a un clarificador artículo de otra de las participantes en este volumen, Anne Berger: «Petite histoire paradoxale des études dites de "genre" en France» (2008).

Queremos dar a conocer igualmente las nuevas iniciativas en apoyo a la investigación con perspectiva de género que se han llevado a cabo en Francia, fundamentalmente con la creación en 2012 del GIS Institut du Genre, por iniciativa del Institut de Sciences Humaines et Sociales del CNRS (Centre National de la Recherche Scientifique). Ligado a numerosas instituciones y equipos de investigación comprometidos en la investigación sobre el género, su objetivo es incentivar la investigación en Francia sobre el género y las sexualidades. Anne Berger, que participa igualmente en este volumen, no solo colaboró en su creación, sino que lo dirigió en los primeros años de su andadura, y le dio el impulso fructífero que ha conseguido en la actualidad.

$\mathrm{Si}$, como decíamos anteriormente, las políticas ministeriales se hacen progresivamente eco de las cuestiones de género, la universidad española no se ha quedado a la zaga, sino que, más bien, ha sido pionera e impulsora de la conciencia de género. Las Unidades de Igualdad y los diferentes Planes de Igualdad constituidos en la mayor parte de las universidades españolas, e incluso la creación de nuevos vicerrectorados, como el Vicerrectorado de Responsabilidad Social, Inclusión e Igualdad, en nuestra Universidad de Alicante, dicen mucho sobre la responsabilidad y el compromiso social de nuestra universidad.

Las diferentes políticas universitarias repercuten positivamente en la investigación en torno al género. Son ya numerosos los Institutos y Centros de investigación sobre la mujer, la igualdad y el género. Si en los nuevos grados la perspectiva de género se incentiva actualmente, debemos decir, haciendo el balance que el título del volumen ofrece, que la Universidad de 
Alicante, y precisamente los estudios literarios desde los que parte el presente volumen, ha sido pionera en la conciencia de la necesidad de visibilizar la aportación real de la mujer, y concretamente la mujer escritora en el mundo de la cultura. La antigua licenciatura de Filología francesa ofrecía ya en los últimos años del pasado siglo una asignatura anual «Escritoras francesas en la historia de la literatura», de carácter optativo pero que, gracias a la elección de materias de libre configuración que los anteriores planes de estudios posibilitaban -y que lamentablemente se ha perdido- permitían a alumnado de diferentes estudios cursar la misma, como de hecho ocurría, con gran satisfacción nuestra al comprobar el interés que la misma despertaba. Dicha asignatura se convirtió en obligatoria para nuestro alumnado de Filología francesa con el nombre de «Literatura francesa y género», incorporando esta nueva perspectiva. Nos queda la satisfacción de que diferentes generaciones de nuestros estudios universitarios han tenido acceso desde hace más de veinte años a una realidad que se ha obviado a lo largo de las épocas, cual es el verdadero papel que las escritoras, y en nuestro caso las escritoras francesas, han desempeñado en los diferentes siglos en el ámbito cultural e intelectual, así como las diferentes causas y vías por las que se han velado su existencia y producción. En definitiva, mantenemos la satisfacción de aportar una visión más justa de la historia literaria, la de nuestros escritores, pero también de nuestras escritoras. La obra que aparecerá próximamente en el mercado dirigida por Martine Reid: Pour une autre histoire des femmes en littérature, y que ella misma avanza en este volumen, da cuenta y es prueba precisamente de esta necesidad.

Sin esta formación, sin este conocimiento previo de una realidad literaria innegable, difícil sería fomentar investigaciones futuras sobre escritoras y género. De nuestro paso por las aulas universitarias surgió la vocación investigadora en literatura y género de otra de las participantes en este volumen,

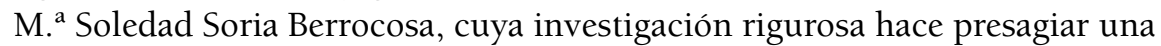
sólida tesis doctoral.

Complementando la formación universitaria, nos planteamos en 1997 la realización de Seminarios de investigación en este ámbito. Así, el Seminario Mujer-Literatura, de carácter bienal, ha disfrutado, bajo la dirección de Ángeles Sirvent, de la presencia de numerosas investigadoras y escritoras como Hélène Cixous, Annie Leclerc, Anne Berger, Martine Reid, Brigitte Diaz, Michèle 
Ramond, Mireille Vincent-Cassis, Dominique Sigaud, Denise Desautels, Suzan Van-Dijk, pero también de un gran número de investigadoras españolas en Literatura y género, ya sea literatura española, catalana, árabe, inglesa o francesa como Marina Mayoral, Carmen Ruiz, M. ${ }^{a}$ Jesús Rubiera, Miriam Tei, Carme Riera, Helena Establier, Carmen Alemany, Silvia Caporale, Teresa Gómez, Claude Benoît, Àngels Santa, Lydia Vázquez, Manuela Ledesma, Brigitte Leguen, Montserrat Serrano, Carmen Marrero, Meri Torrás, Alicia Puleo, Alicia Ramos, Ángeles de la Concha, Montserrat Palau, Mercedes Arriaga, Doina Popa-Liseanu, Encarnación Medina, Marta Segarra, Nieves Ibeas... citadas únicamente según el orden de participación de todas ellas. A ellas uniremos la colaboración de compañeros y compañeras como José Luis Arráez, Amelia Peral, participantes también en este volumen, Josefina Bueno, M. ${ }^{a}$ Carmen Ramón, Maribel Corbí, Ángeles Llorca, M. ${ }^{a}$ Carmen Serrano, María Badiola, Víctor Domínguez, Michele de Sadeleer, Elena Sandakova, Maribel Peñalver, Elisa Lamsfus, M. ${ }^{a}$ Soledad Soria, citados igualmente según el orden de participación en ellos. A todas y a todos agradecemos, en este balance sobre el estado de la investigación en Literatura francesa y género, su contribución al espacio de reflexión crítica de las diferentes temáticas del Seminario.

Uno de estos Seminarios se convirtió en 2011 en un coloquio internacional «Les femmes écrivains en France au XVIII ${ }^{\mathrm{e}}$ siècle. Nouvelles approches», cuyos resultados fueron publicados en la editorial Honoré Champion (2016).

La dilatada trayectoria potenciando los estudios y la investigación en literatura francesa y género determinó la creación en el seno del Instituto Universitario de Investigación en Estudios de Género de la Universidad de Alicante, del Grupo de investigación EEFG (Escritoras de Expresión Francesa y Género) del que forman parte distintos investigadores e investigadoras nacionales y extranjeras; entre otros, diferentes participantes en este volumen como José Luis Arráez, Brigitte Leguen, Encarnación Medina, Maribel Peñalver, Marta Segarra, M. ${ }^{a}$ Soledad Soria y la firmante de esta introducción, Ángeles Sirvent.

Un balance de las investigaciones en Literatura francesa y género no podía quedar completo sin dar cuenta, precisamente, de la investigación expuesta en los diferentes coloquios de la Asociación que aglutina a todas y todos los investigadores sobre los estudios franceses en la universidad española. 
Hemos constatado el progresivo aumento de la investigación sobre nuestras escritoras francesas, aun cuando muchas de estas investigaciones se refieren a aspectos temáticos y formales de la obra literaria. Del mismo modo, y aunque en mucha menor medida, las investigaciones sobre teoría de género se hacen presentes en el último decenio.

El interés patente en visibilizar a estas escritoras de nuestra historia literaria por parte de nuestro colectivo investigador ha conllevado que, desde hace años, las publicaciones sobre literatura francesa y género que resultan de nuestros coloquios representen aproximadamente -y afortunadamente- la mitad de los estudios literarios del volumen; y todo ello sin contar las investigaciones que contemplan a la mujer como objeto literario, es decir como personaje o como tema literario.

Queremos poner de relieve que, todavía, la mayor parte de las investigaciones de dichos coloquios en el ámbito objeto de este monográfico, ha sido llevada a cabo por mujeres, y por ello felicitamos, por su contribución, a aquellos compañeros que son conscientes de que falta mucho por hacer en la configuración de una completa y justa historia de la literatura.

Resulta imposible en el marco de este balance, a modo de introducción, sobre el estado actual de la investigación en literatura francesa y género, aludir a todas estas investigaciones. Ofrecemos pues así en anexo un completo Repertorio en donde se han extraído pacientemente las publicaciones en este ámbito. El lector, la lectora, podrán obtener, no tanto la información sobre las publicaciones llevadas a cabo sobre una autora en particular-que puede conocerse ya por medios informáticos- sino también una visión exacta de la evolución de nuestras investigaciones, los aspectos en que mayormente se ha incidido, y comprobar que no solo las escritoras de nuestra época reciente han gozado de la atención de la crítica, y no solo nuestras escritoras francesas, sino las de los diferentes países de expresión francesa.

Debemos decir que en todos nuestros coloquios se han llevado a cabo investigaciones directamente ligadas a literatura francesa y género -salvo en el IV Coloquio de la APFFUE-, y que todas los textos referenciados están en línea -salvo, de momento, el XXII Coloquio- con lo que son fácilmente accesibles para toda persona interesada en esta investigación.

Los estudios literarios con perspectiva de género son pues ya considerables afortunadamente. Nuestro objetivo al plantear este volumen es llevar a 
cabo un balance crítico y dar a conocer el estado actual de la investigación literaria con perspectiva de género, tanto en Francia como en España, centrándonos en el ámbito de la literatura de expresión francesa, y proponer nuevas vías, nuevos retos en la investigación. Al mismo tiempo pretendemos reflexionar en torno a las diferentes líneas de investigación en este ámbito con mayor pujanza en el momento actual.

A través de su artículo «Pour une autre histoire des femmes en littérature», Martine Reid nos anuncia en este volumen la próxima aparición, en la primavera del 2020, de una importante investigación colectiva llevada a cabo a lo largo de varios años, y que ha tenido como objetivo el ofrecer una amplia historia cultural de la literatura de expresión francesa que da cuenta de la presencia evidente en ella, desde la Edad Media hasta la actualidad, de las mujeres y de sus obras, y que Reid presenta en este artículo.

Siguiendo la estela de sus interesantes aportaciones en Des Femmes en littérature (2010), en donde ya enunciaba las carencias de diferentes historias de la literatura desde Gustave Lanson, la misoginia que revelaban, y ponía de relieve el instrumento de poder que estas representan por cuanto son la base de la enseñanza de nuestro alumnado, el equipo de investigadoras que Martine Reid ha liderado pretende suplir estas carencias otorgando a la mujer en la historia de la cultura su verdadera existencia y aportación.

Reid presenta los objetivos de esta nueva historia de la literatura, que no desatiende las aportaciones de la sociología, la filosofía o la antropología cultural; en definitiva, toda vía de acceso a la vida literaria de las diferentes épocas. En su artículo, Reid alude a las diferentes denominaciones que la mujer escritora ha ido recibiendo en Francia, reflexiona sobre ciertos a priori instalados en la historia literaria, como son la identificación -casi reducciónde la mujer a la novela y a la constitución misma del género, a la tradición que asigna la novela sentimental al género femenino, y al idealismo concedido a la mujer frente al realismo masculino.

Si todavía se necesitan muchos estudios para situar a la mujer escritora en el digno lugar que le corresponde en la historia literaria, mucho mayor desconocimiento se posee de la intervención de la mujer en la prensa y con mayor motivo su presencia en la prensa anterior al siglo XIX. Tras subrayar que incluso el propio término «journaliste» ha poseído una acepción masculina en diversos diccionarios hasta el siglo XX, y poner de relieve el estado

Feminismo/s 34, diciembre 2019, pp. 13-42 
actual de las publicaciones en este ámbito, las investigaciones que M. ${ }^{a}$ Soledad Soria está llevando a cabo -parte de las cuales se ofrece en el artículo de este monográfico: «Invisibles, oubliées, émancipées: les femmes journalistes françaises avant l'heure»-, están permitiendo sacar a la luz la participación y producción femenina en las diversas modalidades que han ido conformando a lo largo de la historia lo que conocemos actualmente como prensa. Desde la contribución de la duquesa de Longueville y la participación asidua de Suzanne de Nervèze en las denominadas «mazarinades» del siglo XVII, Soria va dando cuenta de diferentes mujeres que en los siglos XVII y XVIII van a conformar la historia del periodismo femenino, e incluso del periodismo político femenino ya en la transición al siglo XVIII.

La Sra. Soria pone igualmente de relieve la creación misma de periódicos femeninos y feministas en el s. XVIII y la participación de las mujeres en la dirección de los mismos, mujeres que se convirtieron también en muchos casos en redactoras habituales, abriendo el camino a las escritoras que se implicaron de forma constante en el periodismo desde el siglo XIX.

En el ámbito de la literatura francesa y sin abandonar la perspectiva de género, Marta Segarra nos presenta su artículo «Por una genealogía de la literatura lesbiana francesa», ofreciendo un balance y nuevas propuestas relativas a esta línea de investigación. La hipótesis formulada por la autora es, como indica en su título, la existencia de una genealogía de la literatura lesbiana francesa. Mediante textos escritos por cinco mujeres de principios del siglo XX, aunque sin excluir reflexiones de autoría masculina, la autora demuestra la forma en que el líquido (el agua, además de otros fluidos) se convierte en un elemento textual y metafórico que entreteje los textos de estas cinco escritoras: Renée Vivien, Natalie Barney, Lucie Delarue-Mardrus, Jeanne Galzy y Colette. Segarra se apoya, por una parte, en las nociones de «existence lesbienne»y de «continuum lesbien» de Adrienne Rich, que permiten dar cuenta de una extensa gama de relaciones mantenidas entre mujeres como movimientos de resistencia a la opresión patriarcal, esto es, relaciones homo-afectivas u homosociales. Por otra parte, el concepto de «homosexualidad primaria» o «el primer cuerpo a cuerpo de la madre» de Luce Irigaray servirá igualmente a la autora de soporte para defender este tipo de relación homoerótica que sólo la escritura literaria permite evidenciar. 
Para la investigadora, la «literatura lesbiana» sería aquella que, escrita por mujeres, aunque sin excluir a los hombres, se centra en sus relaciones afectivas y homoeróticas, atendiendo tanto al contenido como a la forma. Si tenemos en cuenta que estas mujeres habrían inventado nuevos modos de representar esta temática, es en la forma donde mejor estamparían el sello de la subjetividad.

Una vez asentadas las bases del marco teórico, Segarra descifra la especificidad de estos textos entre los años 20 y 40 del siglo XX. El elemento líquido se convierte, no solo en la metáfora de las relaciones homoeróticas entre mujeres, sino en la cristalización textual de la fluidez sensual de sus cuerpos, sobrepasando todo tipo de binarismo masculino-femenino y heterosexual-homosexual.

Segarra legitima una especifidad propia de dicha escritura, como es el uso recurrente de la homofonía, ya conocida en francés, entre «mère» $\mathrm{y}$ «mer», metáfora que asociaría el mar a la feminidad materna, además de sexualizar el significado de ambos términos. Explorando estos textos, la autora consigue mostrar la fluidificación de las identidades sexuales susceptibles de ver modificado constantemente su género.

Anne Berger nos presenta su artículo titulado «Un nuevo cuerpo amoroso: escribir el cuerpo en tiempos del MLF». La autora recuerda, de entrada, la duda ontológica que Freud se planteó, dejando sin resolver, a propósito del alma de la mujer: «¿Qué es lo que desea la mujer?» Precisamente la mujer, subraya Berger, como sujeto de deseo, constituyó un tema central en la producción literaria de las escritoras del siglo XX. La investigadora, tras reconocer el papel desempeñado por el psicoanálisis en la invención del «sujeto que desea», y con la finalidad de asentar su marco teórico, expone los antecedentes que motivaron, en las escritoras de la década del MLF, un nuevo enfoque del cuerpo, como fueron, entre otros, los textos de Sand, Vivien o Duras, sin olvidar Le Deuxième Sexe de Beauvoir; la filósofa, y en la línea de Freud, priorizará la exploración de la sexualidad en la formación del sujeto «mujer». La pregunta que Freud dejó sin resolver encontrará ahora su respuesta, señala Berger, precisamente en los textos de mujeres del siglo XX, los más importantes de la época del MLF, y que explora en el presente artículo. Se trata de escritoras cuya escritura del cuerpo se hace explosiva, tales como: Wittig, Cixous, Cardinal, Leclerc, Viviane Forrester, Jeanne Hyvrard o Chawaf. En 
estos, las autoras se reconocen como sujetos de deseo, permitiéndose, en este sentido, abordar libremente la sexualidad, como categoría de la experiencia occidental moderna, desde un desapego que refuta la literatura erótica escrita por hombres. Berger, a través de estos escritos eróticos o pornográficos, establece una asociación intrínseca entre el paso al acto homosexual y el paso al acto literario que permitirá mostrar sin tapujos una posición libidinal activa, una homosexualidad exenta de prejuicios y estereotipos alejados de la heteronormatividad. En esta línea, muestra cómo el deseo de la mujer se inscribe en la lengua de estas escritoras que exploran toda una paleta de experiencias carnales que no se limita únicamente a la homosexualidad; se trata de un auto-erotismo en femenino que presenta un «nuevo cuerpo amoroso», un «cuerpo erogeneizado». Este cuerpo permite interpretar y desvelar las fantasías eróticas, latentes en la memoria de la mujer, pero conservando y desvelando los estigmas de su inconsciente individual y también colectivo, y por tanto en su dimensión histórica. En su análisis discursivo, Berger desencripta la lengua de estas escritoras, y explica que, además de relaciones homosexuales, esta escritura de mujeres apela a diversos recursos propios del cuerpo, como son la maternidad, la menstruación, la masturbación, transcribiendo así un cuerpo erógeno en femenino en todo su potencial inestable e incierto que se reinventa constantemente por el placer que desata. Este cuerpo explosivo, propone Berger, puede ser leído ahora en estos textos, como la anticipación de la deconstrucción queer del cuerpo y sus placeres.

Tras ciertas reflexiones sobre la autoficción frente a la novela autobiográfica y demás manifestaciones de la escritura del yo, y tras aludir a las técnicas de fragmentación del texto, del papel del inconsciente o de los saltos de memoria, Brigitte Leguen nos ofrece el recorrido, la evolución de este tipo de ficción en las escritoras de expresión francesa desde el inicio del presente siglo -a pesar de que alguna de ellas, como Ernaux, han manifestado su resistencia a verse incluidas en el término de autoficción-, y sin olvidar textos significativos precursores en nuestras escritoras del siglo XX.

En su artículo «Autofiction versus écriture de soi chez les écrivaines françaises contemporaines» Leguen incide en la intención manifiesta en muchas de estas producciones literarias de poner de relieve el cuerpo y la sexualidad, el erotismo y la intimidad en todos sus grados, rayando en la sordidez o en la pornografía, la violencia del lenguaje, sacudiendo los yugos

Feminismo/s 34, diciembre 2019, pp. 13-42 
y los estereotipos femeninos. Al mismo tiempo Leguen alude a la relación que se observa entre manifestaciones de la autoficción y ciertos síntomas que caracterizan a la posmodernidad.

Textos de Angot, Cusset, Delaume, Arcan... o la «autoficción teórica» de Despentes recorren el artículo de Leguen en este balance de la autoficción con nombre de mujer.

Dos textos contemplan la investigación sobre las escritoras de expresión francesa y la Shoá. El primero de ellos es el correspondiente a la investigación llevada a cabo por José Luis Arráez, y titulado: «Los relatos testimoniales de mujeres francesas judías durante la Shoá. Estado de la cuestión entre el final de la guerra y finales de siglo». En el marco de la literatura testimonial, el autor examina el número de aportaciones de las mujeres francesas judías, aun recordando testimonios de autoría masculina, explicando la forma en que ha evolucionado su participación en este tipo de literatura. Atendiendo a factores de índole histórico, ideológico o sociológico, el autor detalla, de manera exhaustiva, las publicaciones de diferentes escritoras, entre el final de la guerra y el fin del milenio.

Arráez constata una especificidad patente en el proceso de deshumanización que sufre, frente al hombre, la mujer judía en tanto que mujer, un atentado a su femininad, aun cuando el engranaje estructural de exterminación estaría exento, en su origen, de una política de género; situación específica de la mujer que los estudios de género han permitido poner de relieve. Desde los primeros relatos testimoniales de la Shoá al finalizar la guerra, el investigador demuestra el silenciamiento al que fueron sometidos estos relatos al ser escritos por mujeres. Tres generaciones darán cuenta de los relatos testimoniales que el autor ha clasificado: la primera generación, desde 1945 hasta finales de los 60, cuyos relatos, caracterizados por la espontaneidad en su escritura, manifestarán una solidaridad entre las mujeres, generándose las llamadas «familias de substitución». Arráez lamenta la falta de atención prestada a la voz de estas mujeres judías escritoras por parte de los movimientos feministas franceses, en pleno auge, que se interesaban principalmente por cuestiones de otra índole, tales como la sexualidad o el aborto.

En la segunda década, de los 70 y los 80, aparecerán antologías editadas por hombres donde la voz de la mujer tiene cabida hasta tal punto que se duplicarán las publicaciones de mujeres respecto de la década precedente. 
La labor llevada a cabo por historiadores, pero también de historiadoras, será determinante para romper con la reclusión social de las víctimas. El autor incide en las razones del aumento de publicaciones; entre otras, por la influencia del feminismo francés de mayo del 68. Si bien ya en esta época surgirán estudios de género aplicados a la Shoá, tanto en EEUU, Alemania, Gran Bretaña e Israel, por ejemplo, Francia no desarrollará una perspectiva de género, en este sentido, según el autor hasta la década de los 90.

En cuanto a la tercera época, de los años 90, los relatos testimoniales contribuirán al llamado «travail de mémoire» de la Shoá, siendo ya algunas de las publicaciones dirigidas por mujeres, aunque los estudios de género de este período no favorecen en gran medida a dicho aumento.

La perspectiva de género en estas investigaciones es el objeto precisamente de la contribución de Amelia Peral en este volumen, complementando así el estudio que le precede. Siguiendo, además, la perspectiva de género, como marco común al presente monográfico, Amelia Peral nos adentra en la literatura transgeneracional de la Shoá en su artículo titulado «Perspectiva de género en la literatura de la Shoá: una escritura transgeneracional». La autora pone de relieve de entrada el interés ya mostrado en los estudios de género, desde los años ochenta hasta la actualidad, por el papel incuestionable que desempeñará la mujer judía durante el genocidio nazi.

Destaca numerosas aportaciones bibliográficas de diversas investigadoras americanas, alemanas, israelíes, inglesas y francesas, que, desde una perspectiva histórica, constituyen un jalón esencial en cuanto a las investigaciones de género sobre la Shoá. Se trata de especialistas que han puesto de relieve el papel desempeñado por las mujeres en contraposición a testimonios de hombres en cambio incuestionables. De estos testimonios de mujeres, se evidencia una política de exterminio en función, entre otras, del sexo. Peral Crespo se centra en diferentes obras narrativas de mujeres de la primera, segunda, pero en particular de la tercera generación de la memoria de la Shoá, dando cuenta del silenciamiento al que muchas de estas voces han sido sometidas.

Además de reflexionar sobre las experiencias vividas por estas mujeres, la autora muestra la forma en que la escritura les permitirá salir del silencio. En este sentido, Peral no dudará en subrayar el concepto de «solidaridad», empleado por muchas de ellas, mucho más afianzado entre mujeres que entre hombres, si se tienen en cuenta estos testimonios.

Feminismo/s 34, diciembre 2019, pp. 13-42 
La investigadora señala que, desde finales del 2000, existe una línea de trabajo que se centra en estudiar, desde una perspectiva literaria, los textos de escritoras de la Shoá de expresión francesa. Narrados en primera persona, estos exploran la relación de las autoras con la figura materna, tal y como algunas de las autobiografías han puesto de manifiesto en la escritura transgeneracional. Al analizar algunos de sus textos, Peral Crespo reconoce esta escritura como un legado imprescindible, siendo generador de una resiliencia de la supervivencia que perdure en la memoria. Algunas escritoras de expresión francesa, tales como Vivianne Forrester, Sarah Kofman, Francine Christophe o Catherine Cusset, comparten características propias de la escritura transgeneracional de la Shoá. Además del ensayo, relatar, desde la ficción, la relación con la madre, será un tema central que las colmará del anhelo impidiendo reducirlas al silencio.

Encarnación Medina, en «Ecofeminismo e interculturalidad», lleva a cabo, en la línea de los objetivos de este monográfico, una reflexión crítica sobre una de las corrientes más en pujanza en el momento actual, cual es el ecofeminismo, término creado por Françoise d'Eaubonne en 1974, y, tras dar cuenta del estado actual de los estudios en este ámbito, incide en la estrecha relación de los mismos con la interculturalidad, en la línea ya expresada por Alicia Puleo.

Medina no se limita a poner de relieve la aportación fundamental de dicha investigadora en estos estudios sino que alude a diferentes estudios críticos llevados a cabo tanto en Francia como en diferentes países de los diversos continentes, dejando patente el interés internacional por los mismos, la desvalorización todavía de la mujer, muy ligada a la falta de conciencia ética global, y el camino que es necesario recorrer para lograr una ética intercultural y de género que debe ser contemplada necesariamente también desde la oferta política y en la que la propia Annie Leclerc ha insistido, como se observa en el bello epílogo de este monográfico.

Hemos querido recuperar la palabra de Annie Leclerc, tras años dormida entre nosotros, para rendir un homenaje merecido a esta escritora que, junto a Hélène Cixous, ha sido una referencia y un impulso fundamentales en el movimiento de las mujeres desde los años setenta. Cierra así nuestro monográfico el «Testimonio», como había querido denominarlo Annie Leclerc, vivido en primera persona, de estos decisivos decenios. 


\section{REFERENCIAS BIBLIOGRÁFICAS}

Berger, Anne. «Petite histoire paradoxale des études dites de "genre" en France», Le Français aujourd'hui, 163, Genre, sexisme et féminisme, Paris, Armand Colin, 2008. https://www.cairn.info/revue-le-francais-aujourd-hui-2008-4page-83.htm

Sirvent, Ángeles, M. ${ }^{a}$ Isabel Corbí y M. ${ }^{a}$ Ángeles Llorca (dir.), Femmes auteurs du dix-huitième siècle. Nouvelles approches critiques. Paris: Honoré Champion, 2016. 


\section{ANEXO \\ Escritoras francesas y género en los Coloquios de la Asociación de Francesistas de la Universidad Española ${ }^{1}$}

Aludimos únicamente a las investigaciones sobre las escritoras de expresión francesa y aquellas en que se pone de relieve la condición de la mujer y el género. No hacemos alusión a aquellos otros capítulos en que se trata a la mujer como personaje en obras de autores masculinos.

Tras la referencia completa de la publicación en donde se recogen las investigaciones, estas se incorporan en el mismo orden de la publicación.

En II Coloquio (Almagro. U. Castilla la Mancha)

Bravo Castillo, Juan (coord.), Actas del II Coloquio sobre los estudios de Filología francesa en la Universidad española (Almagro, 3-5 mayo de 1993). Ediciones de la Universidad de Castilla-La Mancha, 1994.

Badía Cubas, Cristina. «Tradición e innovación en la poesía amorosa de l'École lyonnaise: Maurice Scève, Pernette du Guillet, Louise Labé», 233-238.

Gamoneda Lanza, Amelia. «L'image du corps et l'écriture dans l'oeuvre de Marguerite Duras», 377-382.

En III Coloquio de la APFFUE (U. Pompeu Fabra)

Lafarga, Francisco, Albert Ribas y Mercedes Tricás (coords.). La traducción. Metodología, historia, literatura: ámbito hispanofrancés. Barcelona: PPU, 1995. Ribas Pujol, Albert. «Las traducciones de Mémoires d'Hadrien de Marguerite Yourcenar: regularidades en las divergencias». 49-60.

Fernández Ocampo, Anxo. «Intertextualité et traduction chez Marie de France:

Commentaire à la traduction en galicien du lai du "Laüstic"». 195-202.

En IV Coloquio de la APFFUE (U. de Las Palmas de Gran Canaria):

1. La asociación ha ido modificando a lo largo de los años su denominación y siglas: APFFUE, APFUE, AFUE. Todos los textos están en línea, salvo, de momento, el Coloquio de la Universidad de Jaén. 
En V Coloquio de la APFFUE (U. de Murcia)

sn. Aproximaciones diversas al texto literario (V Coloquio celebrado en la Universidad de Murcia, 20-22 marzo 1996). Murcia: Servicio de Publicaciones Universidad de Murcia, 1996.

Bueno Alonso, Josefina. «Le statut de la femme écrivain au dix-neuvième siècle: Judith Gautier». 53-64.

Ibeas Vuelta, Nieves. «El sujeto poético y la autoridad de la voz femenina en Christine de Pizan». 129-136.

Segarra Montaner, Marta. «Stratégies narratives et identité chez les romancières maghrébines». 215-222.

Millán Muñío, M. a Ángeles, «La lectura como experiencia de género». 325-332. Moltó Hernández, Elena. «Cuando el placer es cortés», 333-342.

Marrero Marrero, M. ${ }^{a}$ del Carmen. «La lectura en el contexto de la Ilustración», 497-506.

VI Coloquio de la APFFUE (U. de Santiago)

García-Sabell, Teresa et alii (coords.), Les Chemins du texte: VI Coloquio da APFUE (Santiago, 19-21 de febreiro de 1997) (2 vol.). Santiago de Compostela: Servicio de Publicaciones Universidade de Santiago de Compostela, 1988.

Bénit, André. «De l'image au texte et du texte à l'image: littérature et cinéma: Suzanne Lilar et André Delvaux». Vol. 1, 61-71.

Bueno Alonso, Josefina. «L'écriture de Leïla Sebbar: croisements textuels et culturels». Vol 1. 85-96.

Durand Guiziou, Marie-Claire. «Système de nomination dans Une liaison parisienne de Marie-Claire Blais». Vol. 1, 183-196.

Ventura, Daniela. «L'Heptaméron de Marguerite de Navarre: un recueil de véritables histoires». Vol. 1, 438-447.

Vicente Pérez, Javier. «L'expression du temps dans L'amant de Marguerite Duras». Vol. 1, 503-516.

VII Coloquio de la APFFUE (U. de Cádiz)

SN, Relaciones culturales entre España, Francia y otros países de lengua francesa (2 vol.). Cádiz: Servicio de publicaciones Universidad de Cádiz, 1999.

Ledesma Pedraz, Manuela. «Présence espagnole dans les études yourcenariennes». Vol. 1, 191-204. 
Romero Alfaro, Elena. «Reflexiones sobre la traducción de la "Relation du voyage d'Espagne" de Madame d'Aulnoy». Vol 1, 447-458.

\section{Coloquio de la APFFUE (U. de Valencia)}

Pujante, Domingo, Elena Real, Dolores Jiménez y Adela Cortijo (coord.). Écrire, traduire et représenter la fête. Valencia: Universitat de València, 2001.

Monleón Domínguez, Ana. «Transgression et ludisme: la Drag Queen». 373-380. Aragón Cobo, Marina. «Françoise Sagan, une sempiternelle fêtarde?». 483-493. Olmo Cazevieille, Françoise. «Claire Brétecher fait la fête aux femmes modernes: ironie et traduction». 769-778.

\section{Coloquio de la APFFUE (Granada)}

Serrano, Montserrat, Lina Avendaño y M. ${ }^{\text {a }}$ Carmen Molina (coords). La philologie française à la croisée de l'an 2000: Panorama linguistique et littéraire. (2 vol.). Granada: Universidad de Granada, 2000.

Avendaño Anguita, Lina. «El fin de siglo de Nathalie Sarraute (1980-1997)». Vol. 1, 47-54.

Benoît, Claude. «Mujer y escritura frente al año 2000: Christine Angot y su libro Linceste». Vol. 1, 63-72.

Cortijo Talavera, Adela. «Un siglo de mujer, una mujer del siglo: Nathalie Sarraute y/o Tachok. Vol. 1, 73-82.

Grijalba Castaños, Covadonga. «Literatura, civilización y sociedad entre dos siglos/milenios (Ferraille de Pascale Roze). Vol. 1, 103-110.

Jover Silvestre, Yolanda B. «Souffrance et liberté dans Une odeur de henné de Cécile Oumhani», Vol. 1, 287-298.

\section{Coloquio de la APFFUE (La Laguna. Tenerife)}

Oliver Frade, José Manuel (coord.). Isla abierta: Estudios franceses en memoria de Alejandro Cioranescu (3 vol.). La Laguna: Servicio de Publicaciones Universidad de La Laguna, 2004.

Arráez Llobregat, José Luis. «Dominique Rolin: un "je” en quête d'un passé; un moi à la rencontre d'un/de l'avenir». Vol. 1, 71-84.

Badía Cubas, Cristina. «La expresión del amor en la poesía de Louise Labé». Vol. $1,135-146$. 
Benoit, Claude. «Amores sáficos y escritura autobiográfica en los albores del s. XX (L. de Pougy, R. Vivien y N. Clifford Barney)». Vol. 1, 179-194.

Blérald-Ndagano, Monique. «Maryse Condé: religion et discours social». Vol. 1, 213-226.

Cuasante Fernández, Elena. «La literatura africana escrita por mujeres: de la novela testimonial a la novela comprometida». Vol. 1, 411-428.

Ortiz Suárez, Adriana - Blanca Liliana Fenoy. «Marguerite Duras: être hors là». Vol. 2, 509-516.

González Menéndez, Lidia. «"Les Terres nouvelles" dans les reportages de Gabrielle Roy». Vol. 2, 599-622.

Marrero Marrero, M. ${ }^{a}$ del Carmen. «La noción de libertad en la escritura femenina francesa del s. XVIII». Vol. 2, 813-824.

Peral Crespo, Amelia. «Du je(u) à l'écriture dans Souffle et La d'Hélène Cixous». Vol. 2. 1009-1022.

Renouprez, Martine. "Gestation et accouchement chez quelques écrivaines belges francophones à partir des années 70». Vol. 3, 1153-1168.

Romero Alfaro, Elena. «El cuento de La belle et la bête de Mme Leprince de Beaumont». Vol. 3, 1187-1200.

Sánchez Hernández, Ángeles. «Estudio de la relación madre-hija en la novela Une femme d'Annie Ernaux». Vol. 3, 1237-1247.

Serrano Mañes, Montserrat. «Un divertissement de salón: le café, joli petit vice des précieuses». Vol. 3, 1293-1304.

Verdegal Cerezo, Joan. «Un siglo de senectud y renovación en el jurado Fémina». Vol. 3, 1459-1476.

\section{Coloquio APFFUE (U. de La Rioja)}

Salinero Cascante, M. ${ }^{a}$ Jesús e Ignacio Iñarrea Las Heras (coords.). El texto como encrucijada. Estudios franceses y francófonos (2 vol.). Logroño: Universidad de La Rioja, 2003.

Salinero Cascante, M. ${ }^{a}$ Jesús. "Le temps au féminin": aproximación a la vida cotidiana femenina a través de los textos medievales». Vol 1, 63-78.

Mendoza Ramos, M. ${ }^{a}$ del Pilar. «El gesto en los "Lais" de Marie de France». Vol 1, 123-136.

Viselli, Sante Arcangelo. «Littérature et peinture dans la fiction romanesque du XVIII ${ }^{e}$ siècle». Vol. 1, 261-276. 
Aubry, Anne. «Larmes, douleur et corps souffrant dans Indiana de George Sand». Vol. I, 311-332.

Cabello Andrés, Nuria. «Mme de Staël y Constant». Vol. 1, 323-340.

Arráez Llobregat, José Luis. «Relecture de ces "paroles de femme" d'Annie Leclerc» Vol. 1, 401-408.

Pérez García, Sonia. «Saveurs et senteurs dans Le blé en herbe de Colette». Vol. 1, 571-580.

Anoll i Vendrell, Lidia. «La fonction des références bibliques dans l'oeuvre romanesque d'Anne Hébert». Vol. 1, 633-646.

Bueno Alonso, Josefina. «Francophonie plurielle: l'expression d'une nouvelle identité culturelle». Vol. 1, 685-696.

Pagán López, Antonia. «Le visible et l'invisible: Moi, Tituba sorcière». Vol. 1, 721-732.

Rinne, Marie Noëlle. «Identités de femmes et espaces géographiques dans la littérature canadienne d'expression française». Vol. 1, 755-766.

Sagarra Martín, Catalina. «L'éternel féminin ou la construction de l'altérité féminine». Vol. 1, 767-780.

Collard, Marie-France. «Images de retour, retour des images: Le chemin symbolique dans Le Vice-Consul». Vol. 2, 43-58.

Jorge Chaparro, M. ${ }^{a}$ del Carmen. «Étude lexicale de certains termes en relation avec les sentiments dans les Lais de Marie de France». Vol. 2, 309-318.

\section{Coloquio de la APFFUE (U. Alicante)}

Sirvent Ramos, Ángeles (coord.). Espacio y texto en la cultura francesa (3 vol.) Alicante: Servicio de Publicaciones de la Universidad de Alicante, 2006.

Álvarez Prendes, Emma. «Le dépaysement (ou le non-espace) chez Duras». Vol. 1, 57-68.

Benoit, Claude. «L'espace imaginaire de l'amour lesbien dans l'œuvre poétique de R. Vivien». Vol. 1, 69-80.

De Diego Martínez, Rosa - Lydia Vázquez. «Mujeres maximalistas». Vol. 1, 95-104. Ledesma Pedraz, Manuela. «Marguerite Yourcenar ou le refoulement du féminin». Vol. 1, 119-136.

Martínez Muñoz, Mado. «El discurso lésbico de Renée Vivien en Une femme m'apparut como subversión del discurso patriarcal». Vol. 1, 147-154.

Muñoz Zielinski, M. ${ }^{a}$ Teresa. "Universo real, universo imaginario, espacio real, espacio imaginario: Colette, Sido y La maison de Claudine». Vol. 1, 169-178. 
Ninanne, Dominique. "Trois femmes hors quatre murs, trois femmes en quête d'histoire: une lecture de Claire Lacombe, Berty Albrecht et Charlotte de Michèle Fabian». Vol. 1, 179-198.

Pagán López, Antonia. «L'espace et le mot dans l'imaginaire mernissien». Vol. 1, 199-216.

Peral Crespo, Amelia. «Las mujeres en el espacio velado de la escritura: una realidad "involuntaria"». Vol. 1, 217-226.

González Dopazo, Olaya. «Gabrielle Roy: personajes en busca de su espacio». Vol. 1, 389-398.

Pardo Jiménez, Pedro. «El espacio de la escritura en los relatos cortos de Béatrix Beck». Vol. 1, 399-412.

Serrano Belmonte, Carmen. «El espacio natural en Pluie et vent sur Télumé Miracle». Vol. 1, 429-436.

Cortijo Talavera, Adela. «Un imaginaire marin das l'oeuvre de Marie Darrieussecq». Vol. 2, 775-798.

Díaz Martínez-Falero, Miguel Ángel. «La insularidad como espacio de muerte en Un homme obscur de Marguerite Yourcenar». Vol. 2, 811-828.

Ruiz Álvarez, Rafael. «De l'espace littéraire à l'espace filmique». Vol. 3, 1893-1908.

XIII Coloquio de la APFUE (U. Oviedo)

Bango de la Campa, Flor M. ${ }^{a}$, Antonio Niembro y Emma Álvarez Prendes (coords.). Intertexto y Polifonía. Estudios en Homenaje a M. ${ }^{a}$ Aurora Aragón (2 vol.). Oviedo: Servicio de Publicaciones de la U. de Oviedo, 2008.

Andron, Marie-Pierre, «Mon cher grand fou... Lettres à Marcel Carbotte 1947-

1979 ou la mise en jeu du 'je' de l'écrivain chez Gabrielle Roy». Vol. 1, 93-100. Aubry, Anne. «Forme et signification du motif religieux dans Angélique de Montbrun de Laure Conan et Indiana de George Sand». Vol. 1, 117-124.

Avendaño Anguita, Lina. Vol. 1. «Enfance de Nathalie Sarraute: une voix scindée». Vol. 1, 125-132.

Benoit, Claude. «Marguerite Yourcenar y la manía de la escritura». Vol. 1, 157-164. González Dopazo, Olaya. «Gabrielle Roy: escritora inmigrante frente a personajes inmigrantes». Vol 1, 291-296.

González Menéndez, Lidia. «S'exiler de l'exil: Rue Deschambault de Gabrielle Roy». Vol. 1, 305-312.

Guijarro García, Rafael. «La narration imposible: le bal de T. Beach dans Le Ravissement de Lol V. Stein». Vol. 1, 327-334. 
López Martínez, Marina. «Le tissage de textes dans un polar au féminin: Légole de la phobie de Brigitte Aubert». Vol. 1, 405-412.

Martins, Celina. «Lintertextualité à la manière d'Ananda Devi. Poétique de la réécriture et du palimpseste». Vol. 1, 453-460.

Ninane, Dominique. «»Cherchant les Indes, nous dûmes inventer l'Amérique», ou comment la mise en scène d'Amphitryon de Kleist aboutit à une adaptation de Michèle Fabien». Vol. 1. 517-523.

Peral Crespo, Amelia. «Flytemnnestre’à «Samsonge». Histoire d'une réécriture ou le jeu de l'imbrication chez Cixous». Vol. 1, 525-532.

Raventós Barangé, Anna. «El palimpsesto kleistiano: sobre las recreaciones de Jean Grosjean y Fabienne Pasquet». Vol. 1. 565-573.

Ribelles Hellín, Norma. «L'Amant de Marguerite Duras: littérature et cinéma, traduction et adaptation». Vol. 1, 575-582.

Viselli, Sante Arcangelo. «Intertexte et fiction narrative chez Madame de Tencin». Vol. 1, 715-722.

González Hernández, Ana Teresa. «Un estudio de los marcadores polifónicos en Les Demoiselles sous les éberniers de Suzanne Prou». Vol. 2, 833-840.

Moscardó Rius, Joana. «Isabelle Eberhardt: nomadismo e identidad». Vol. 2, 1061-1068.

\section{Coloquio de la APFUE (U. Valladolid)}

Ramos Gómez, M. ${ }^{a}$ Teresa y Catherine Desprès Caubrière (coord.). Percepción y realidad. Estudios Francófonos. Valladolid: Servicio de Publicaciones Universidad de Valladolid, 2007.

Avendaño Anguita, Lina. «L'énonciation de la perception chez Nathalie Sarraute», 409-415.

Boilève-Guerlet, Annick. «Réalités féminines: Le quotidien au temps de Christine de Pizan», 443-451.

Marrero Marrero, Carmen. «La caricatura de Mme du Deffand: Una manera de percibir al otro», 663-670.

Peral Crespo, Amelia. «Le pouvoir des mots dans l'écriture féminine: Les paroles que ma mère m'a versées ont percé ma réalité». 713-720.

Ribelles Hellín, Norma. «Les couleurs de L'entrave: Étude chromatique du roman de Colette», 765-773.

Rodríguez Navarro, M. ${ }^{a}$ Victoria. «La percepción del día y la noche en Louise Labé», 783-790. 
Ruiz Álvarez, Rafael. «Aimer. Être aimé. Se perdre, de Annie Ernaux», 799-805. Sánchez Fernández, Ángeles. «Una percepción del universo literario de Fatou Diome», 833-841.

Soler, Ana. «La perception tamisée d'une douloureuse réalité: Entendez-vous dans les montagnes... de Maissa Bey», 877-884.

Gala Guillén, Begoña. «Hiroshima mon amour, el relato de un espejismo», 1045-1052.

XV Coloquio de la APFUE (Huelva)

Bonnet, Dominique, M. ${ }^{a}$ José Chaves García y Nadia Duchêene (coord.). Littérature, langages et arts. Rencontres et création. Huelva: Universidad de Huelva, 2007.

Badiola Dorronsoro, María. «Arte y búsqueda espiritual en Magnus, de Sylvie Germain».sp.

Boidard Boisson, M. ${ }^{a}$ Cristina y Flavia Aragón Ronsano. Ce pays dont je meurs de Fawzia et L'année de l'éclipse de Fatima Ben Mansour: deux rencontres créatives sur l'immigration maghrébine. sp.

Ballestín Cucala, Cristina. «Claude Cahun o la desnaturalización del sexo». sp. García Casado, Margarita. «De l'amour divin à l'amour humain: La femme de Job d'Andrée Chedid». sp.

Lozano Sampedro, M. ${ }^{a}$ Teresa. «El dibujo y la pintura en un cuento iniciático de George Sand: Le château de Pictordu». sp.

De Agustín Guijarro, Javier. «L'expérience musicale devenue discours: Les Variations Goldberg de Nancy Huston». sp.

Cortijo Talavera, Adela. «La bande dessinée française au féminin». sp.

García Gandul, Auxiliadora. «La mirada cotidiana como fuente de escritura en La vie extérieure d'Annie Ernaux». sp.

Ramón Díaz, M. ${ }^{a}$ del Carmen. «Le voyage des filles à travers l'oeuvre ségurienne». sp.

García Pradas, Ramón. «Louise Labé, débitrice de l'amour médiéval ou créatrice d'une nouvelle conception de l'amour au XVI ${ }^{e}$ siècle». sp.

Pujante González, Domingo. «Méditations esthétiques o la pintura hecha poesía (encuentros y desencuentros entre Apollinaire y Marie Laurencin)». sp.

Soler Pérez, Ana. «Portraits de femmes dans l'oeuvre beyenne Cette fille-là». sp. González Dopazo, Olaya. "Yves Thériault y Gabrielle Roy: el tratamiento de la mujer esquimal en un mundo de hombres». sp. 
XVI Coloquio de la APFUE (U. Lleida)

Santa Bañeres, Àngels et alii (coord.). Texto y sociedad en las letras francesas y francófonas. Lleida: Universitat de Lleida, 2009.

Sirvent Ramos, Ángeles. «La crítica social y política de Olympe de Gouges y Manon Roland». 187-199.

Solé i Castells, Cristina. «L'espace dans les romans de jeunesse de George Sand», 220-229.

Gonzalo Santos, Tomás. «Les Beaux Messieurs de Bois-Doré de George Sand, una reescritura del Quijote». 230-238.

Solà Solé, Pere. «Le Rendez-vous des étranges de Elsa Triolet». 364-374.

Corbí Sáez, M. ${ }^{a}$ Isabel. «Le deuxième sexe de Simone de Beauvoir, encore d'actualité». 384-394.

Noeno Caraballo, M. ${ }^{a}$ Teresa. «Joyce Mansour, la mujer maldita», 407-418.

Ballestín Cucala, Cristina. «La experiencia surrealista de Bona de Mandiargues: Bonaventure», 419-431.

Peral Crespo, Amelia. «Écrire les silences de l'être sombrant dans la nuit (Annie Ernaux et Marie Chaix)». 463-473.

Sánchez Hernández, Ángeles. «El "etnotexto" de Annie Ernaux como fotografía de la realidad». 474-483.

Cortijo Talavera, Adela. «Una sociedad animalizada en el Zoo de Marie Darrieussecq». 553-567.

González Dopazo, Olaya. «Peuples de Canada de Gabrielle Roy: una nueva perspectiva de la figura del inmigrante en tierras canadienses». 758-767.

Anoll i Vendrell, Lidia. «Pour quoi, pour qui Emma hurlait-elle? Au sujet du Livre d’Emma, de Marie-Célie Agnan».

Castellani, Jean Pierre. «La réception de Camus en Algérie: Maïssa Bey, lectrice d'Albert Camus». 803-810.

Watts, Françoise. «Textes de l'urgence: Maissa Bey et la société algérienne». 811-821.

\section{Coloquio de la APFUE (U. Salamanca)}

Gonzalo Santos, Tomás et alii (Coord.). Texto, género y discurso en el ámbito francófono. Salamanca: Ediciones Universidad de Salamanca, 2016.

Merino García, M. ${ }^{a}$ Manuela. «La nouvelle du XVII ${ }^{e}$ siècle, une technique en évolution: Anaxandre et La princesse de Montpensier». 377-390. 
Lozano Sampedro, M. ${ }^{a}$ Teresa. «La tierra o el eterno renacer: Le Marteau rouge de George Sand». 419-431.

Ballestín Cucala, Cristina. "La "Salomé" de Claude Cahun». 435-448.

Peral Crespo, Amelia. «De L'amant de Mireille Sorgue à Lamante de François Solesmes: désir de l'être entre deux mains s'écrivant ou l'entre-deux désirs d'être s'écrivant».

Ninane, Dominique. «Ladieu à la «pièce bien faite» dans l'oeuvre de Michèle Fabien». 501-508.

Benoit,Claude. «Lucidité et pessimisme dans l'oeuvre de Natacha de Pontcharra». 521-526.

Pich Ponce, Eva. «La présence du corps dans l'écriture de Marie-Claire Blais». 551-560.

González Dopazo, Olaya. «Les Lettres chinoises de Ying Chen: dos voces para una escritura mestiza». 561-570.

Jover Silvestre, Yolanda B. «Solitude et violences dans Plus loin que la nuit de Cécile Oumhani». 599-606.

Anoll i Vendrell, Lidia. «Seul ce qui brûle, de Christiane Singer: réécriture d'un conte de Marguerite de Navarre». 781-792.

Sánchez Hernández, Ángeles. «Alain Corneau, interprète cinématographique du discours littéraire d'Amélie Nothomb». 845-856.

XVIII Coloquio de la APFUE (U. Jaume I)

Sanz Gil, Mercedes y Joan Manuel Verdegal Cerezo (Coord.). Construcción de identidades y cultura del debate en los estudios en lengua francesa. Castellón: Universitat Jaume I, 2011.

Agustín Guijarro, Javier de. «Enfance et écriture féminine en Afrique: trois cas de mise en texte d'un imaginaire». 257-270.

Gascía, Mar. «La fable contrariée: La Cathédrale d'Ananda Devi, de l'écrit à l'écran». 326-339.

Incardona, Janine. Le pèlerinage amoureux de Guénelie dans Les Angoysses douloureuses d'Hélisenne de Crenne». 360-374.

Llop Gacía. Lluna Llecha. Rêve, étrangeté et identité dans Les chambres de bois, d'Anne Hébert». 375-388.

López Martínez, Marina. «Double je de Sylvie Granotier, un polar d'ombres et de doubles». 389-396. 
Lozano Sampedro, M. ${ }^{a}$ Teresa. «Identidad y Naturaleza en un relato fantástico de George Sand: Laura, voyage dans le cristal». 397-410.

Mallart Brussosa, Myriam. «L'exclusion dans deux romans de Léonora Miano». 421-432.

Sanz Pérez, Celia. «Sophie est Sophie». 497-509.

Mangada Cañas. Beatriz. «Nuevas identidades francófonas: recorrido por una de las voces de la interculturalidad literaria de expresión francesa [Wei-Wei]». 693-702.

Mata Barreiro, Carmen. «Écrivaines francophones noires: voix de mémoires blessées et passeures de cultures». 703-712.

Saiz Cerreda, M. ${ }^{a}$ del Pilar. «Un espace, deux langues: la négociation d'une identité francophone chez Gabrielle Roy». 757-765.

XIX Coloquio de la APFUE (U. Complutense. Madrid)

Losada, José Manuel (coord.). Tiempo, texto e imagen. Temps, texte et image. Madrid: Universidad Complutense, 2010.

Aubry, Anne. La 'venue à l'écriture' de Laure Conan dans Angéline de Montbrun». 267-278.

Bénit, André. Un voyage dans le temps, le temps d'un voyage: Anya de Clémentine Faik-Nzuji». 291-302.

Figuerola Cabrol. Maria Carme. «Reproduire le temps de l'attente chez Michèle Desbordes». 335-346.

Elena García, Margarita. «Espace et temps dans la trilogie La Passion et les hommes de Nicole Verschoore». 387-396.

González Alarcón, Isabel Esther. «Voces femeninas en Une si longue lettre de Mariama Bâ». 419-430.

Herrero Cecilia, Juan. «La vida y la muerte frente a la inmortalidad en Tous les hommes sont mortels (1946) de Simone de Beauvoir». 455-468.

Lozano Sampedro, M. ${ }^{a}$ Teresa. «Tiempo, espacio e imagen en La Femme rompue de Simone de Beauvoir». 503-516.

Montaner Sánchez, Lucía. «El tiempo (a través de Bourrasque de Hélène Lenoir)». 563-572.

Peral Crespo, Amelia. «Mémoire et autofiction dans l'oeuvre de Catherine Cusset». 587-600.

Pich Ponce, Eva. «Le temps et l'écriture du chaos dans Soifs de Marie-Claire Blais». 601-610. 
Pujante González, Domingo. «Les Inconvenances de Martine Roffinella: caricatures du présent et nostalgie du passé (ou l'écriture de la faille)». 623-632.

Reboul, Anne-Marie. «La conception du temps dans Le Grand Jamais d'Elsa Triolet». 645-654).

Robustillo Bayón, Eva. «Tiempo y novela policiaca: el caso de Sous les vents de Neptune de Fred Vargas». 655-662.

Sánchez Hernández, Ángeles. «Imagen autobiográfica y tiempo sociológico en Les Années de Annie Ernaux». 673-684.

Gutiérrez Viñayo, Félix César. «Images du temps dans L'Empreinte de l'Ange de Nancy Huston» 1079-1090.

XX Coloquio de la APFUE (U. Zaragoza)

Bermejo Larrea, Esperanza, Corcuera Manso, Juan Fidel y Julián Muela Exquerra (coord.). Comunicación y escrituras. Communication et écritures. En torno a la lingüistica y la literatura francesas. Zaragoza: Prensas Universitarias de Zaragoza, 2012.

Lojo Tizón, M. a del Carmen. «La temática decadente en Monsieur Vénus (1884) de Rachilde» 315-322.

López-Santibáñez, M. ${ }^{a}$ Isabel. «La expresión del vacío en L'usage de la photo, de Annie Ernaux». 323-328.

Montaner Sánchez, Lucía. «Las técnicas de escritura en Hélène Lenoir». 389-396. Montes Villar, Luisa. "Adelaida Blázquez, una escritura de "l'entre deux"». 397-406.

Pagán López, Antonia. «Écritures au service de l'art: Mme de Staël et George Sand». 417-432.

Pich Ponce, Eva. «Le corps et l'Autre: la configuration du moi dans Le Sourd dans la Ville de Marie-Claire Blais». 443-450.

Sánchez Hernández, Ángeles. «Mémoire et histoire à travers trois sensibilités au féminin». 481-494.

Solé i Castells, Cristina. «Clara Malraux et la société de son temps». 503-510.

XXI Coloquio de la APFUE (U. Barcelona-U. Autònoma Barcelona)

Catena, Angels et alii. Les mondes du français. XXI Colloque de l'Asociación de Profesores de Francés de la Universidad Española. Barcelona: Ediciones de la Universitat Autònoma de Barcelona i la Universitat de Barcelona, 2013. 
Marti, Alexandra. «Recuperación de la memoria histórica en la película francesa "La Rafle" de Roselyne Bosch». 101-107.

Cremades Cano, Isaac David «Maryse Condé y Gisèle Pineau: de la antillanidad a un criollismo en femenino». 141-151.

Figuerola Cabrol, Maria Carme. «Le maître des âmes de Irène Némirovsky o cómo trazar un retrato del desarraigo». 176-188.

Ledesma Pedraz, Manuela. «La liberté libre à l'épreuve de la transculturation chez Malika Mokeddem». 209-219.

Lojo Tizón, M. ${ }^{a}$ del Carmen. «Figuras masculinas en algunas novelas de Rachilde», 220-228.

Lozano Sampedro, M. ${ }^{a}$ Teresa. «La Reine Coax de George Sand: el género de lo maravilloso y la inversión de sus figuras». 237-247.

Malingret, Laurence. «L'écriture féminine maghrébine: d'une rive à l'autre, d'une langue à l'autre». 248-254.

Montaner Sánchez, Lucía. «(S')écrire en français: la langue française et l'expérience d'étrangeté chez Hélène Lenoir». 272-276.

Montes Villar, Luisa. «Trayectorias marginales: exilio y escritura en la obra de Adelaida Blázquez». 277-288.

Pich Ponce, Eva. «Marie-Claire Blais, Margaret Atwood et les États-Unis: une certaine vision de la littérature». 298-305.

XXII Coloquio de la APFUE (U. Jaén)

Merino García, M. ${ }^{a}$ Manuela et alii. L'Appréciation langagière de la Nature: Le natural, le texte et l'artifice, Jaén: Publicaciones de la Universidad de Jaén. 2017.

Verna Haize, Christine. «La fauvette rythme les saisons et les acacias évoquent l'amitié amoureuse au jardín de Mlle de Scudéry». 89-98.

Lafarga Maduell, Francisco. «Expresión y sentimiento del paisaje en el relato del viaje a España de la Duquesa de Abrantes». 105-112.

Bermúdez, Medina, M. ${ }^{a}$ Dolores. «J'ai fait de la Nature le décor de ma volonté. Les Hors Nature de Rachilde». 143-152.

Lojo Tizón, M. ${ }^{a}$ del Carmen. «Naturaleza y artificio en la novela de Rachilde (1860-1953)». 193-198.

Lozano Sampedro, M. ${ }^{a}$ Teresa. «La voz de la naturaleza y el género de lo maravilloso en algunos relatos breves de George Sand». 199-211.

Andrade Boue, Pilar. «Naturaleza y mujer en Un barrage contre le Pacifique y Withe material». 293-302. 
Benoit, Claude. «Linterprétation de la nature chez Marguerite Yourcenar». 311-319.

Fouchard, Flavie. «La naissance du jour de Colette: Laube et la naissance d'une poétique». 327-334.

López Múgica, Montserrat. «Corinna Bille: de la naturaleza a la obra literaria». 355-361.

Montes Villar, Luisa. «Paisajes de una tierra llamada lengua y de un sueño llamado España. cruce de miradas entre Michel del Castillo y Adelaida Blázquez». 373-379.

Novotná, Sárka. «Les apparences illusoires du mimétisme et d'autres artifices ontologiques: l'esquisse de la dialectique de la nature et de l'artificiel dans Kamouraska d'Anne Hébert». 381-390.

Cremades Cano, Isaac David. «Poética del espacio y paisaje subjetivo en En attendant la montée des eaux de Maryse Condé». 453-460.

El Bakali, Naufal. «La reconquête des lieux et des origines du personnage-femme dans le récit de Marrakech Lumière d'exil de Rajae Benchemsi». 461-466.

González Alarcón, Isabel Esther. «Image de la femme algérienne vue à travers les yeux d'Assia Djébar». 477-485.

Labra Cenitagoya, Ana I., «Lumière sur lumière: fonctions de la métaphore lumineuse dans la production narrative de Mohamed Dib et Assia Djébar». 487-496.

Pich Ponce, Eva. «Paradis ou enfer: la présence de la Nature dans l'oeuvre romanesque de Marie Claire Blais». 513-520.

Robustillo Bayón, Eva. «Naturaleza, ciudad y crimen en las novelas del comisario Adamsberg de Fred Vargas». 541-545.

Urquhart, Steven. «Champagne (2008) de Monique Proulx: une représentation sublime de la Nature». 571-582.

\section{Coloquio de la APFUE (U. Alcalá)}

Labra Cenitagoya, Ana I. et alii. Crisis, ¿fracaso o reto? Crises, échec ou défi?. Alcalá de Henares: Servicio de Publicaciones Universidad de Alcalá, 2016.

Coca Méndez, Beatriz. «L'expression de la fidélité envers les valeurs noires dans Résidente privilégiée de María Casares». 90-100.

Fouchard, Flavie. «La fin de Chéri de Colette: mourrir après la guerre (1914-18)». 148-157. 
Humières, Catherine d'. «Résolution des crises et visées éducatives chez la Comtesse de Ségur et Trilby». 193-207.

Jover Silvestre, Yolanda B. «Crise identitaire et rébellion. L'enfance d'un écrivain dans Le coeur à rire et à pleurer de Maryse Condé». 221-231.

Lojo Tizón, M. ${ }^{a}$ Carmen. «La figura de Rachilde durante la crisis literaria española de principios de siglo». 238-246.

Lozano Sampedro. M. ${ }^{\text {a }}$ Teresa. «La crisis de la adolescencia y el sentido de lo maravilloso en George Sand: Le nuage rose». 247-258.

Pich Ponce, Eva. «Face à la mort: l'importance du deuil dans Fleurs de Crachat de Catherine Mavrikakis». 299-306.

Romeral Rosel, Francisca. «Psychogenèse d'une crise familiale: La fille démantelée de Jacqueline Harpman». 319-332.

\section{Coloquio AFUE (U. Almería)}

Cantón Rodríguez, Loreto et alii. Metáforas de la luz. Métaphores de la lumière. APFUE, 2017.

Figuerola Cabrol, Maria Carme. «A la luz de George Sand: La petite Fadette». 210-219.

González Hernández, Ana Teresa. «Pas pleurer de Lydie Salvayre: une écriture d'ombre et de lumière». 220-229.

Lozano Sampedro, M. ${ }^{a}$ Teresa. «La luz y el imaginario mineral en George Sand. Análisis aproximativo de algunos relatos». 258-267.

Robustillo, Bayón, Eva. «De la oscuridad a la luz en Sans feu ni lieu, de Fred Vargas». 326-331.

Férriz Núñez, María. «Estudio de la luz en los relatos de Cécile Oumhani». 421-429.

Ledesma Pedraz, Manuela. «Assia Djebar ou le désir d'Icare au féminin». 430-437. Rouane Soupault, Isabelle. «Reflets et miroitements: Les confessions de Saint Augustin dans le roman de Claude Pujade-Renaud, Dans l'ombre de la lumière». 446-456.

Hernández Guerrero, M. ${ }^{a}$ José. «Las poetas de Quebec traducidas en España». 483-494.

\section{Coloquio AFUE (U. Politècnica València)}

López Santiago, Mercedes et alii. Palabras e imaginarios del agua. Les mots et les imaginaires de l'eau. València: Editorial Universitat Politècnica València, 2017. 
Corbí Sáez, M. ${ }^{a}$ Isabel. «Le symbolisme de la mer dans Les Plages d'Agnès Varda au miroir de la littérature». 13-20.

Cortijo Talavera, Adela. «L'eau vivante et l'eau morte dans l'univers féminin du cinéma tunisien: la mer dans La Saison des hommes (2000) de Moufida Tlatli et la salle de bains dans Les Secrets (2009) de Raja Amari». 21-26.

Haderbache, Ahmed. «Prise de parole et quête de liberté: les espaces de l'eau dans Aïcha de Yamina Benguigui». 35-40.

Monleón Domínguez, Ana. «Traversées migratoires: Des étoiles (2013) de Dyana Gaye». 49-56.

Pujante, González, Domingo. «Rites et rythmes de l'eau et du désir dans Mossane de Safi Faye». 57-64.

Tamarit Vallés, Inmaculada. «La recréation du hammam dans l'univers féminin de Karin Albou», 65-72.

Aceituno Martínez, Eduardo. «El agua como símbolo y componente del espacio en los relatos de Maryse Condé». 285-290.

Benoit Morinière, Claude. «Images de l'eau dans l'œuvre yourcenarienne». 337-344.

Bonnet, Dominique. «Maylis de Kerangal: une écriture au coeur de la mer». 345-350.

Corsin, Julie. «Silvia Baron-Supervielle ou la poétique de l'eau». 367-374.

Cremades Cano, Isaac David. «Eau et mémoire chez Marie-Célie Agnan». 375-380.

González Hernández, Ana Teresa. «La femme au colt 45: un parcours dans l'imaginaire aquatique de Marie Redonnet». 425-432.

Lojo Tizón, M. ${ }^{a}$ del Carmen. «El imaginario del agua en Rachilde (1860-1953)». 475-480.

Lozano Sampedro, M. ${ }^{a}$ Teresa. «La palabra del agua en la narrativa de George Sand: Ce que dit le ruisseau». 489-496.

Marcotte, Sophie. «Le calme après la tempête: le pouvoir symbolique de l'eau dans l'œuvre de Gabrielle Roy». 505-512.

Pich Ponce, Eva. «Entre Eros et Thanatos: l'imaginaire de l'eau dans Lî̀le de la Merci, d'Élise Turcotte». 567-572.

Rodríguez Navarro, María Victoria. "L'Amour à deux visages: el agua en Pernette du Guillet como reflejo del diálogo amoroso». 573-582.

Sánchez Hernández, Ángeles. «Les associations thématiques du motif de l'eau dans un roman québécois: HKPQ de Michèle Plomer». 589-596.

Szyman, Alexandra, «Les mots et les imaginaires de l'eau dans l'œuvre de Simone Schwarz-Bart». 605-612. 